\title{
Un nuevo Método para Mejorar el Proceso de Producción de Acido Bórico
}

\author{
Orlando J. Domínguez, Emilio M. Serrano, Jorge E. Flores y Raquel L. Michel \\ Universidad Nacional de Salta, Facultad de Ingeniería, Consejo de Investigación, Instituto de \\ Beneficio de Minerales, Avenida Bolivia 5150, (4400) Salta-Argentina (email: \\ orlando@unsa.edu.ar, eserrano@unsa.edu.ar, jeflores@unsa.edu.ar, rmichel@unsa.edu.ar,)
}

Recibido Mar. 16, 2010; Aceptado May. 14, 2010; Versión Final recibida Jul. 07, 2010

\begin{abstract}
Resumen
En este trabajo se propone una modificación al proceso tradicional para obtener ácido bórico, con el agregado fraccionado del ácido lixiviante, para lograr un producto altamente soluble, como el pentaborato de sodio. Con esto se consigue la disolución del mineral en frío con menor cantidad de reactivo lixiviante, ahorrando así energía. Al líquido clarificado se le disminuye el pH logrando así la precipitación del ácido bórico. La ganga se agota con diferentes etapas de precipitación y filtración, con sus consiguientes lavados, mejorando el rendimiento del proceso. El ácido bórico producido presenta características, tales como textura y pureza, comparables con el obtenido por los procesos tradicionales.
\end{abstract}

\section{A New Method to Improve the Production Process of Boric Acid}

\begin{abstract}
This paper proposes a modification to the traditional process for obtaining boric acid with the addition of acid leaching split, to get a highly soluble product, as sodium pentaborate. In this way the mineral can be dissolved without heating, decreasing the amount of leaching reagent, thus saving energy. The $\mathrm{pH}$ of the clarified liquid is decreased leading to the precipitation of boric acid. The gangue runs out at different stages of precipitation and filtration, with its successive washes, improving process performance. The produced boric acid has characteristics, such as texture and purity, comparable with that obtained by traditional processes.
\end{abstract}

Keywords: borates, boric acid, ulexite, sodium pentaborate, leaching 


\section{INTRODUCCION}

Desde el punto de vista tecnológico, el bórax y el ácido bórico son los principales productos refinados del boro, tanto por su aplicación (usos) como por su empleo como materia prima para la obtención de otros productos: anhídrido bórico, tetra y pentaborato de amonio, perborato, pentaborato y octoborato de sodio, boratos de cinc y otros (Marcus, 1997).

Salta es el primer productor y exportador de boratos de Argentina y uno de los principales a nivel mundial (Secretaría de Minería de Salta, 2009).

La ulexita, $\mathrm{Na}_{2} \mathrm{O} .2 \mathrm{CaO} .5 \mathrm{~B}_{2} \mathrm{O}_{3} \cdot 16 \mathrm{H}_{2} \mathrm{O}$ (Garret, 1998) es un borato doble de calcio y sodio. Si bien parte de la producción se exporta como preconcentrado, una cantidad sustancial se procesa en la provincia con la finalidad de producir ácido bórico.

El ácido bórico tradicionalmente se obtiene por la reacción de un ácido mineral con un mineral de borato, siendo la más difundida la reacción del ácido sulfúrico con un mineral de ulexita, debido a su abundancia en la Puna Salteña (Alonso, 1998), según se indica en la reacción (1):

$\mathrm{Na}_{2} \mathrm{O} .2 \mathrm{CaO} .5 \mathrm{~B}_{2} \mathrm{O}_{3} \cdot 16 \mathrm{H}_{2} \mathrm{O}+3 \mathrm{H}_{2} \mathrm{SO}_{4} \Leftrightarrow 2 \mathrm{Ca} \mathrm{SO} 4.2 \mathrm{H}_{2} \mathrm{O}(\mathrm{s})+\mathrm{Na}_{2} \mathrm{SO}_{4}(\mathrm{ac})+10 \mathrm{H}_{3} \mathrm{BO}_{3}$

Esta reacción se realiza a una temperatura entre 70 y $80^{\circ} \mathrm{C}$, a los efectos de solubilizar el $\mathrm{H}_{3} \mathrm{BO}_{3}$ producido, debido a que su solubilidad disminuye fuertemente con la temperatura. En el proceso tradicional, por enfriamiento, se logra la cristalización del mismo (Flores, 2004). Esto significa un gasto de energía para calentar y termostatizar, además de un gasto de equipamiento para mantener su aislamiento. La otra variable importante es el $\mathrm{pH}$ que se debe mantener entre 1 y 2 , para garantizar un agotamiento del mineral, lo que implica un exceso de ácido sulfúrico, con un alto riesgo de contaminación ácida en el caso de evacuación de los barros o eventuales derrames de efluentes, atentando contra el medio ambiente.

En la Figura 1 se muestra el proceso tradicional para la obtención de ácido bórico que utilizan las plantas actuales que operan en Argentina, "sin apreciables modificaciones de lo indicado por Garret (1998)".

Varios grupos de investigadores estudiaron la cinética de la disolución de ulexita en soluciones de ácido sulfúrico en función de la temperatura, concentración de ácido, granulometría, velocidad de agitación y concentración de masa en el proceso de disolución (Imamutdinova, 1969; Tunç et al., 1999; 2001).

Debido a los problemas ambientales ocasionados por el manejo de un ácido fuerte como el sulfúrico y como una manera de disminuir el gasto en energía calórica, se propone en este trabajo una modificación al proceso de producción de ácido bórico mediante el agregado fraccionado de ácido sulfúrico.

En el proceso alternativo propuesto se plantea el agregado fraccionado de ácido sulfúrico según la siguiente reacción (2):

$\mathrm{Na}_{2} \mathrm{O} .2 \mathrm{CaO} .5 \mathrm{~B}_{2} \mathrm{O}_{3} \cdot 16 \mathrm{H}_{2} \mathrm{O}+2 \mathrm{H}_{2} \mathrm{SO}_{4} \Leftrightarrow 2 \mathrm{CaSO}_{4}(\mathrm{~s})+2\left[\mathrm{NaB}_{5} \mathrm{O}_{8} .5 \mathrm{H}_{2} \mathrm{O}\right]+8 \mathrm{H}_{2} \mathrm{O}$

De esta manera el pentaborato de sodio obtenido permanece en solución a temperatura ambiente debido a que su solubilidad es mayor que la del ácido bórico (Flores, 2004). El sulfato de calcio sólido se evacua junto con la ganga que acompaña al mineral. De esta manera se dispone de una solución límpida de alto contenido de $\mathrm{B}_{2} \mathrm{O}_{3}$ con lo que se consigue la disolución del mineral en frío con disminución del reactivo lixiviante ahorrando de esta manera energía calórica. 


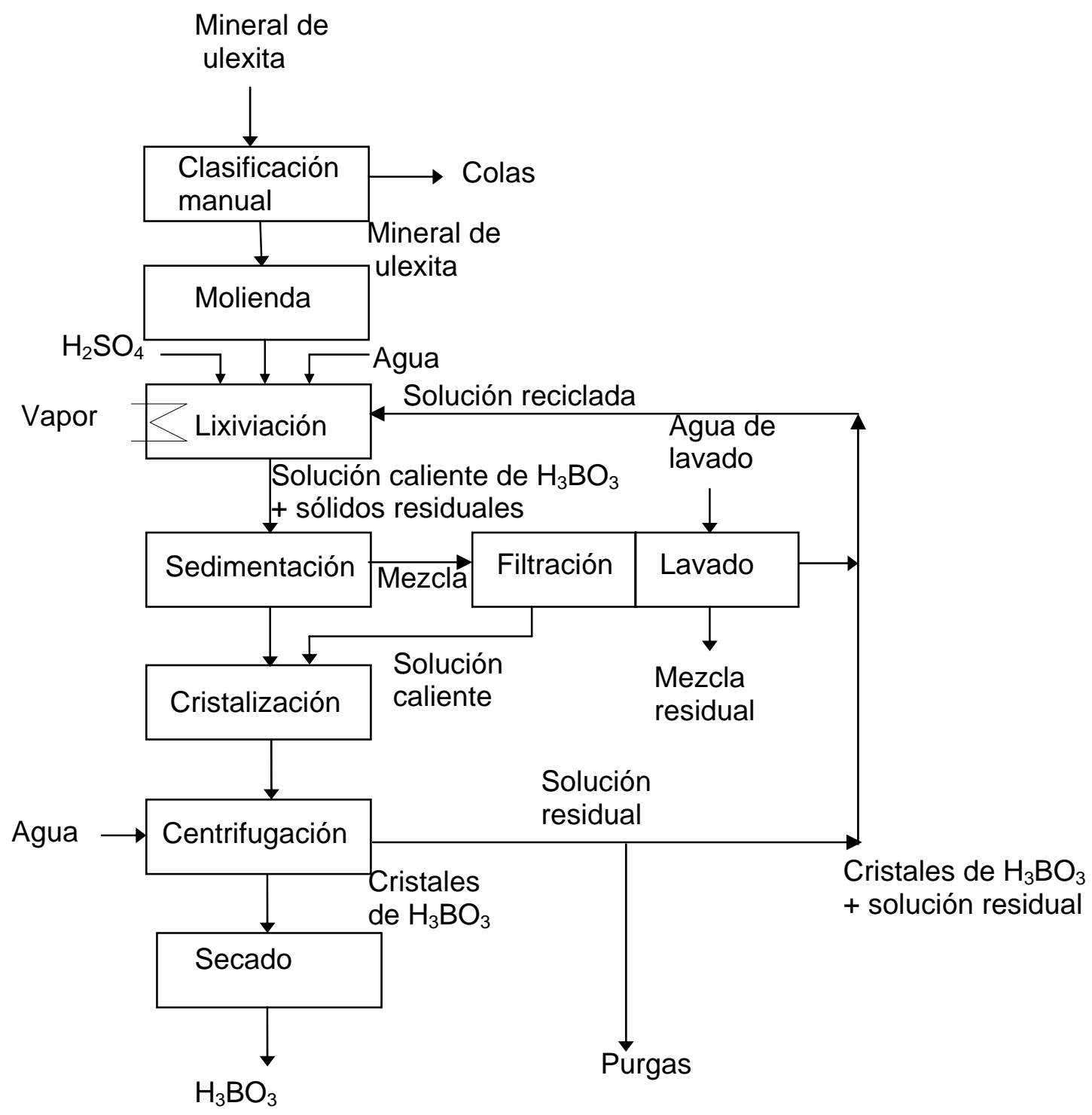

Fig.1: Diagrama del proceso tradicional de producción de Acido Bórico.

\section{TRABAJO EXPERIMENTAL}

Los ensayos experimentales se realizaron a escala Planta Piloto a partir de mineral de ulexita proveniente del Salar Centenario con una ley de $\mathrm{B}_{2} \mathrm{O}_{3}$ del $32 \%$ y molido a malla Mesh $\mathrm{N}^{\circ} 16$ (ASTM), correspondiente a pasante de $1 \mathrm{~mm}$. Se empleó una relación de masa de ulexita a agua madre 1 a 4 expresada en $\mathrm{kg} / \mathrm{l}$.

En la Figura 2 se observa el esquema de producción del proceso alternativo de obtención de ácido bórico seguido en los ensayos experimentales, en cuyo balance de materia se tomó como base una tonelada de mineral de ulexita pura.

El barro está impregnado con un líquido de alto contenido de $\mathrm{B}_{2} \mathrm{O}_{3}$ de igual concentración a la solución, por lo tanto debe ser sometido a una etapa de lavado a los efectos de recuperar el boro. A los efectos de mejorar el rendimiento del proceso, el líquido de lavado se une al líquido sobrenadante de la etapa anterior, agotando el boro del barro. Se ensayaron dos técnicas: una la decantación inducida por floculación y lavado y la otra la filtración y lavado de la torta.

Un posterior agregado del resto del ácido sulfúrico produce la transformación del pentaborato en ácido bórico, según la reacción (3).

$2\left[\mathrm{NaB}_{5} \mathrm{O}_{8} .5 \mathrm{H} 2 \mathrm{O}\right]+4 \mathrm{H}_{2} \mathrm{O}+\mathrm{H}_{2} \mathrm{SO}_{4} \Leftrightarrow 2 \mathrm{Na}_{2} \mathrm{SO}_{4}(\mathrm{~s})+10 \mathrm{H}_{3} \mathrm{BO}_{3}$ 


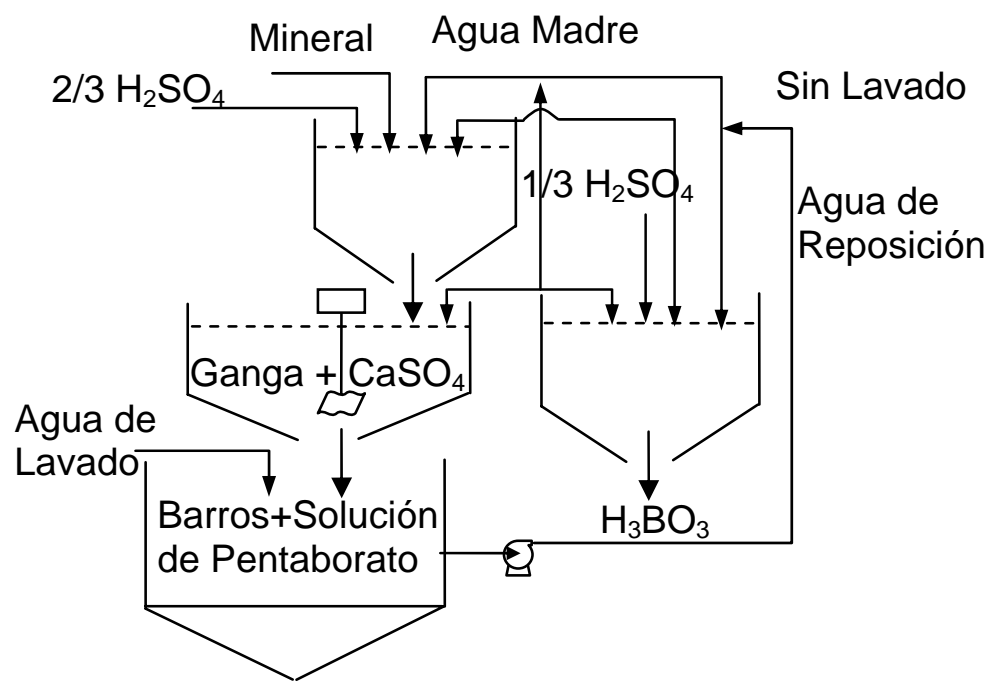

Fig. 2: Alternativa de producción de $\mathrm{H}_{3} \mathrm{BO}_{3}$

El sulfato de sodio continúa en solución eliminándose una fracción del circuito como humedad que acompaña al barro y el resto del líquido se recircula como agua madre hacia la etapa inicial de disolución.

La variable preponderante de la experiencia, es la cantidad de barro producido que depende directamente de la ley del mineral. Este barro está integrado por la ganga y el sulfato de calcio dihidratado, que al aumentar su volumen lleva consigo un mayor volumen de agua con pentaborato disuelto, de allí la necesidad del lavado de los barros generados.

Para evitar las pérdidas de $\mathrm{B}_{2} \mathrm{O}_{3}$, expresadas como $\mathrm{H}_{3} \mathrm{BO}_{3}$, en los barros, surgen de inmediato dos opciones: concentrar el mineral o lavar para recuperar los barros.

La concentración del mineral implica una pérdida al sacrificar una fracción de colas para mejorar la ley de cabeza. Es por ello que se adoptó como variable de estudio el lavado para recuperar el boro.

A medida que disminuye la ley aumenta la cantidad de agua madre, de modo que la concentración de $\mathrm{B}_{2} \mathrm{O}_{3}$, valor constante, disminuye al disminuir la ley, obteniendo un valor de $\mathrm{kg}$ de $\mathrm{B}_{2} \mathrm{O}_{3}$ por metro cúbico de agua madre, cantidad que indicaría que la concentración en el agua que acompaña al barro sería menor. Mientras que la cantidad total de líquidos que acompañan al barro aumenta con la disminución de la ley, lo que implicaría una mayor cantidad de $\mathrm{B}_{2} \mathrm{O}_{3}$ perdido. Si se analiza el efecto total, las pérdidas aumentan con la disminución de la ley, por lo tanto debemos reconocer que este segundo efecto es de mayor incidencia que el primero de dilución.

La rápida disminución del rendimiento exige una recuperación que debe producirse por una extracción del boro disuelto retenido en los huecos del sólido.

Para ello se realizaron ensayos de lavado consistentes en una agitación de los barros precipitados con floculante, de modo que el agua agregada disminuye la concentración del borato y de esta manera se puede lavar hasta en dos etapas sucesivas, obteniéndose en ambas por adición de pequeñas cantidades de ácido, el ácido bórico correspondiente. No hace falta un nuevo agregado de floculante para la precipitación del sólido inerte. 
En el caso de realizarse la recuperación por filtración, este sólido obtenido tiene una alta porosidad de modo que garantiza el llenado de los marcos del filtro, no siendo limitante entonces su superficie.

La comparación entre estas dos últimas alternativas es la operatividad, su inversión y su costo de operación, debido a que la utilización de filtros, que en el caso de no ser equipos automáticos, son muy caros o bien insumen una mayor mano de obra, no presentando este inconveniente la alternativa de limpieza por lavado y decantación, debido que esta última trabaja de forma continua y se regula con la dosificación de floculante en caso de ser necesario.

De los productos obtenidos de los ensayos realizados, se observa que el ácido bórico cristalizado, obtenido del pentaborato de sodio por una sola cristalización ácida tiene un rendimiento del $50,6 \%$, realizando un primer lavado se incrementa a un rendimiento del $73 \%$ y efectuando un segundo lavado, el rendimiento alcanza el $77,4 \%$, de modo que con 2 lavados se obtiene en el proceso el rendimiento indicado.

\section{ANALISIS DE LAS EXPERIENCIAS}

El grado de disgregación del mineral es muy importante, de manera de aumentar la velocidad de reacción, debido a que el ataque con el ácido se realiza en frío, "como lo sugiere Aguilar (2008)". Igual importancia tiene la agitación para lograr un íntimo contacto sólido-líquido.

En la Figura 3 se representa el proceso propuesto de obtención de ácido bórico, a través de un ciclo similar a los termodinámicos, a los efectos de ilustrar el proceso desde su inicio hasta el nuevo ciclo, limitado por las curvas de solubilidad del pentaborato de sodio y las del ácido bórico, que son los productos en tránsito dentro del proceso propuesto.

Se puede trabajar con minerales de ley menores al 30\% para no alcanzar la solubilidad a temperatura de $25^{\circ} \mathrm{C}$. En el caso de tener leyes superiores que podrían aportar mayor concentración, es necesario suministrar una pequeña cantidad de calor Q entre los puntos 2 y 3 del ciclo de la Figura 3, que lo lleva a la zona de no saturación.

Analizando la Figura 3, en el punto 1, vértice inferior izquierdo, se comienza con el agua madre y mineral a $25^{\circ} \mathrm{C}$ y $\mathrm{pH}$ entre 2 y 2,5 que con el agregado de $2 / 3$ de ácido sulfúrico genera un aumento de temperatura de aproximadamente $6^{\circ} \mathrm{C}$, punto indicado como 2, que en el caso de superar la curva de solubilidad de $\mathrm{B}_{2} \mathrm{O}_{3}$ del pentaborato de sodio se entraría en la zona de sobresaturación, por lo que es conveniente suministrar una cantidad de calor Q de manera de aumentar la solubilidad del pentaborato llegando al punto 3. En este punto 3 se alcanza el pH de 5,8 , correspondiente a la solubilidad del pentaborato, luego se induce la floculación y se separa el líquido clarificado del barro sedimentado. El líquido clarificado se pasa a otro recipiente donde se le suministra el $1 / 3$ restante de ácido sulfúrico aumentando la temperatura $3^{\circ} \mathrm{C}$, como consecuencia del agregado del ácido, alcanzándose el punto 4. La disminución de la solubilidad es debida a la transformación del pentaborato en ácido bórico, un enfriamiento posterior ayuda a cristalizar el ácido bórico, cerrando de este modo el ciclo.

Los datos cinéticos entre los puntos 3 y 4 del ciclo de la Figura 3 se siguieron con un pHmetro, siendo el $\mathrm{pH}$ del agua madre inicial de 5,23, inferior al del pentaborato, ésto debido a que las aguas madres tienen un remanente de ácido, dándose por concluida esta etapa cuando el $\mathrm{pH}$ alcanza el valor de 5,8.

Estos datos cinéticos corresponden a una temperatura de $23^{\circ} \mathrm{C}$ y sin exceso apreciable de ácido.

En la Tabla 2 se tabularon los valores cinéticos de la conversión de pentaborato a ácido bórico en frío. Se observa que el agregado de ácido sulfúrico produce una disminución muy rápida del pH, amortiguándose su ascenso hasta llegar al valor final que no es coincidente con los valores conocidos de 4,4 a 4,6, por la presencia de pentaborato de sodio que forma una mezcla reguladora. 


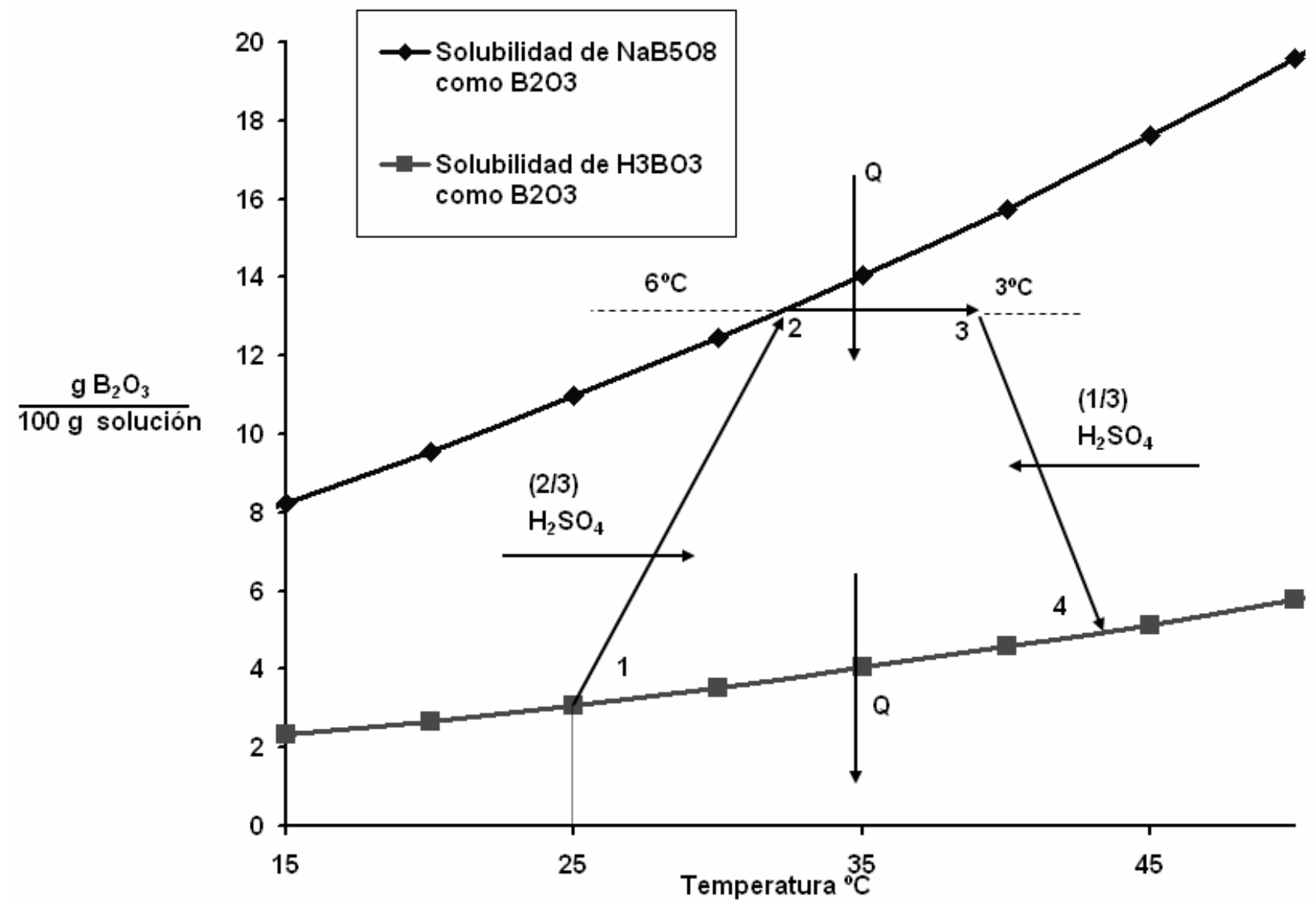

Fig. 3: Representación del proceso propuesto de obtención de ácido bórico.

Se advierte que los datos cinéticos en frío son inferiores a los que se obtienen para un agotamiento total del mineral con ácido sulfúrico en caliente, "como lo muestra Pocoví et al. (2004; 2005)".

Tabla 2: Datos cinéticos de la conversión de pentaboraro a ácido bórico

\begin{tabular}{|c|c|}
\hline Tiempo (minutos) & $\mathrm{pH}$ \\
\hline 0 & 5,23 \\
\hline 6 & 2,80 \\
\hline 13 & 4,90 \\
\hline 29 & 5,31 \\
\hline 52 & 5,50 \\
\hline 65 & 5,58 \\
\hline 78 & 5,70 \\
\hline 91 & 5,71 \\
\hline
\end{tabular}




\section{Sample Name : $\quad$ BD3H3-M1}

$4 k$

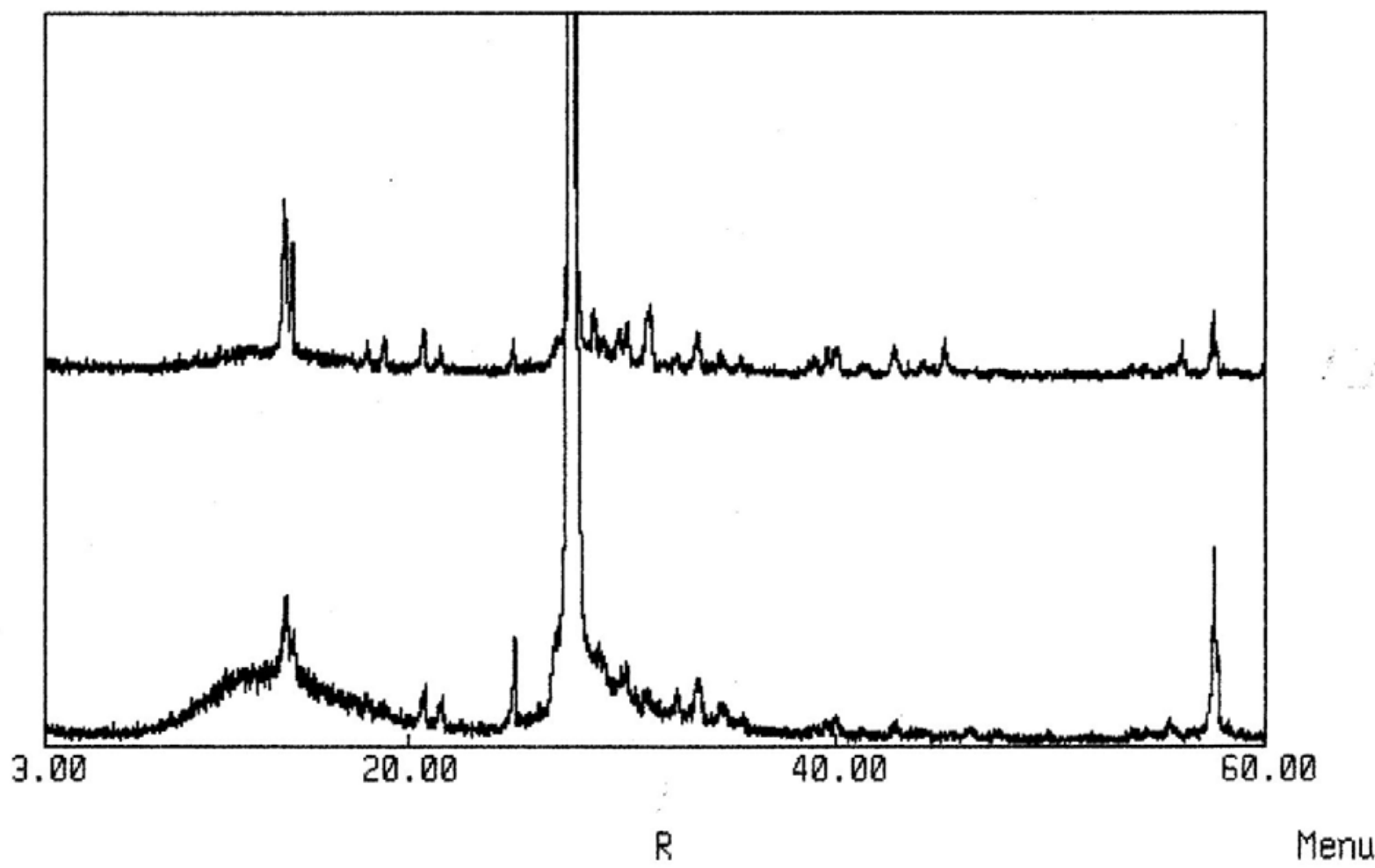

Fig. 4: Análisis de RX del Acido Bórico obtenido.

\section{CONCLUSIONES}

El ácido bórico producido es de calidad comercial similar al obtenido en los procesos convencionales, en cuanto a su pureza. En la Figura 4 se muestra un análisis de $\mathrm{RX}$ del $\mathrm{H}_{3} \mathrm{BO}_{3}$, siendo el superior correspondiente al obtenido con los ensayos de cristalización.

El ahorro de energía de calefacción con vapores provenientes de un generador de vapor, no sólo produce una disminución del costo de inversión y de producción, sino que también disminuye la contaminación ambiental, por la disminución de emisiones de $\mathrm{CO}_{2}$.

Los barros a evacuar presentan una concentración mucho menor de ácido sulfúrico, porque el último agregado de este ácido, se realiza sobre una solución límpida, no acidificando los barros, pudiendo como alternativa utilizar ácidos degradables, como por ejemplo el ácido acético para disminuir el $\mathrm{pH}$.

\section{REFERENCIAS}

Aguilar E. W., Procedimiento para la Obtención de Acido Bórico a partir de Boratos Naturales, Actas de IX Jornadas Argentinas de Tratamiento de Minerales y XVI Reunión Anual de la Asociación Iberoamericana de Enseñanza Superior de la Minería, 259-267, San Juan, 20 al 22 de Octubre (2008).

Alonso, R., Los Boratos de la Puna, Cámara de la Minería de Salta, 129, Salta, Argentina (1998).

Flores H.R. El Beneficio de los Boratos, 130-134, 147-150, 209. Ed. Crisol. Salta, Argentina (2004). 
Garrett D.E., Borates, Handbook of Deposits, Processing, Properties and Use, Academic Press, 360, USA, (1998).

Imamutdinova, V. M.; Kinetics of dissolution of borates in mineral acid solutions, Zh. Prikl. Khim., 40 (11), 2593-2596 (1967).

Marcus, J., Rio Tinto and U.S. Borax Inc., E\& MJ, USA, 24-32 (1997).

Pocoví, R, E. Serrano, J. Flores y L. Kwok, Estudio Cinético Comparado de la Lixiviación de Ulexita con Acido Sulfúrico y Acido Clorhídrico para Producir Acido Bórico, Actas de VII Jornadas Argentinas de Tratamiento de Minerales, SEGEMAR-INTEMIN, 131-138, Buenos Aires, ARGENTINA, 20 al 22 de Octubre (2004).

Pocoví, R, E. Serrano, J. Flores y L. Kwok, Distinción de Dos Etapas en la Reacción de Ulexita con Acido Mineral, Ingeniería Química, 428, 425-430 (2005).

Secretaria de Minería de Salta, Producción minera, Exportaciones de la Provincia de Salta, años 2001-2009, Salta, Argentina, (2009).

Tunç M., Kocakerim, M. M., Yapici, S. e Y.S. Bayrakceken, Dissolution of ulexite in $\mathrm{H}_{2} \mathrm{SO}_{4}$ solution, Hydrometallurgy, 51, 359-370 (1999).

Tunç, M., S. Yapici, M. Kocakerim, and A. Yartasi, The Dissolution Kinetics of Ulexite in Sulphuric Acid Solutions, Chem Biochem. Eng. Q., 15(4), 175-180, (2001). 